\title{
La cochinilla corticícola Matsucoccus feytaudi Duc., 1941 y su papel en el decaimiento de Pinus pinaster Ait. en el Parque Natural de la Sierra de Baza (Granada, Andalucía, España)
}

The role of Matsucoccus feytaudi Duc., 1941 influencing Pinus pinaster Ait. decline in the Natural Park of the Sierra of Baza (Granada, Andalusia, Spain)

Guzmán Álvarez, J.R. ${ }^{*}$; Gómez de Dios, M.A.'; Muñoz Risueño, A. ${ }^{2}$; Alguacil Picón, F. ${ }^{2}$; Gómez Milán, F. ${ }^{2}$; Nebot Sanz, B. ${ }^{3}$; Cobos Aguirre, J.F.J. ${ }^{1}$; Carrasco Gotarredona, A. ${ }^{1}$; Rodríguez Reviriego, S. ${ }^{2}$; Sánchez Callado, F.M.'; Redondo Noval, J.A.'; Ruiz, J.M.'; Sánchez Anguita, A. ${ }^{3}$

\footnotetext{
${ }^{1}$ Dirección General de Medio Natural, Biodiversidad y Espacios Protegidos, CAGPDS; Avda. Manuel Siurot, 50 - 41071 -, Sevilla, España.

${ }^{2}$ Equilibrios Biológicos, Agencia de Medio Ambiente y Agua de Andalucía (AMAYA) Consejería de Agricultura, Ganadería, Pesca y Desarrollo Sostenible (CAGPDS); C/Johan G. Gutenberg, 1 - 41092 -, Sevilla, España.

${ }^{3}$ Delegación Territorial de Granada, CAGPDS: C/ Joaquina Eguaras $n^{\circ} 2$. Edificio "Almanjayar" - 18071, Granada, España.
} 


\title{
Resumen
}

En 2016 se produjo un episodio de decaimiento de gran intensidad sobre los pinares de repoblación de pino resinero (Pinus pinaster) del Parque Natural de la Sierra de Baza. Los primeros indicios de este proceso de mortalidad masivo se habían observado en 2014 y 2015 en Sierra de Baza y Sierra de los Filabres (Granada y Almería), así como en la cara norte de Sierra Nevada. Asociado a este proceso se observó la presencia notable de la cochinilla Matsucoccus feytaudi. Con objeto de dilucidar el papel que este insecto estaba desempeñando en la muerte del arbolado, en febrero de 2017 se colocaron 12 trampas de feromonas desarrolladas por el INRA (Francia) en la Sierra de Baza y 8 trampas adicionales en otras zonas de Andalucía, tanto en rodales procedentes de repoblación como con origen natural, con o sin manifestación de síntomas patentes de decaimiento. Se presentan los resultados derivados del trampeo llevado a cabo durante los meses de febrero a mayo de 2017, los cuales muestran una presencia muy extendida de $M$. feytaudi en la mayor parte de las localizaciones, discutiéndose el rol de este insecto en el episodio de decaimiento. Los resultados no sugieren que este insecto haya actuado como factor desencadenante en este episodio de decaimiento, pero sí puede haber actuado como factor contribuyente que acelera la muerte del arbolado.

Palabras clave: cambio climático, ecosistemas mediterráneos, feromona, Margarodidae, monitoreo, mortalidad de pinar.

\begin{abstract}
A severe forest decline event affecting around 2000 ha of maritime pine plantations (Pinus pinaster) occurred during the summer of 2016 in the Natural Park Sierra de Baza (Granada, Andalusia, Spain). Scattered mortality of pines, but with lower intensity was previously detected in 2014 and 2015 in surroundings areas. Symptoms were uniform and included foliage discolouration and lost, following by the death of the trees, affecting frequently the whole stand. Associated with the dieback, an outbreak of Matsucoccus feytaudi was observed, being considered as a potential causal factor involved in the mortality. Twelve pheromone sampling traps (developed by INRA, France) were set in the month of February 2017 in the area and its surroundings (Natural Park of the Sierra de Baza) to monitor the presence of male insects. Additionally, eight traps were placed in other maritime pine stands (both in plantations and natural-origin areas, and with presence and absence of decline symptoms) to compare the incidence of M. feytaudi. Results from the survey (from February to May 2017) shows that $M$. feytaudi spread across most of the sampled areas, even in stands with healthy forest conditions. The results dodn't suggest that $M$. feytaudi acted as a triggering factor in this decline event; instead, this insect could have act as contributing factor which speeds up the decline process.
\end{abstract}

Keywords: climatic change, Margarodidae, mediterranean ecosystems, monitoring, pheromones, pine dieback. 


\section{Introducción}

Durante julio y agosto de 2016 tuvo lugar un episodio severo de mortalidad de pino negral (Pinus pinaster Ait.) procedentes de repoblación en el Parque Natural de la Sierra de Baza, estimándose en un inicio que la superficie afectada se situaba en torno a 2000 ha (Fig. 1 y Fig. 2). Previamente, a lo largo de 2014 y 2015, había sido registrada por parte del Equipo de Equilibrios Biológicos de la Consejería de Agricultura, Ganadería, Pesca y Desarrollo Sostenible de la Junta de Andalucía la muerte de ejemplares aislados y un estado de debilitamiento apreciable en algunos sectores del Parque Natural.

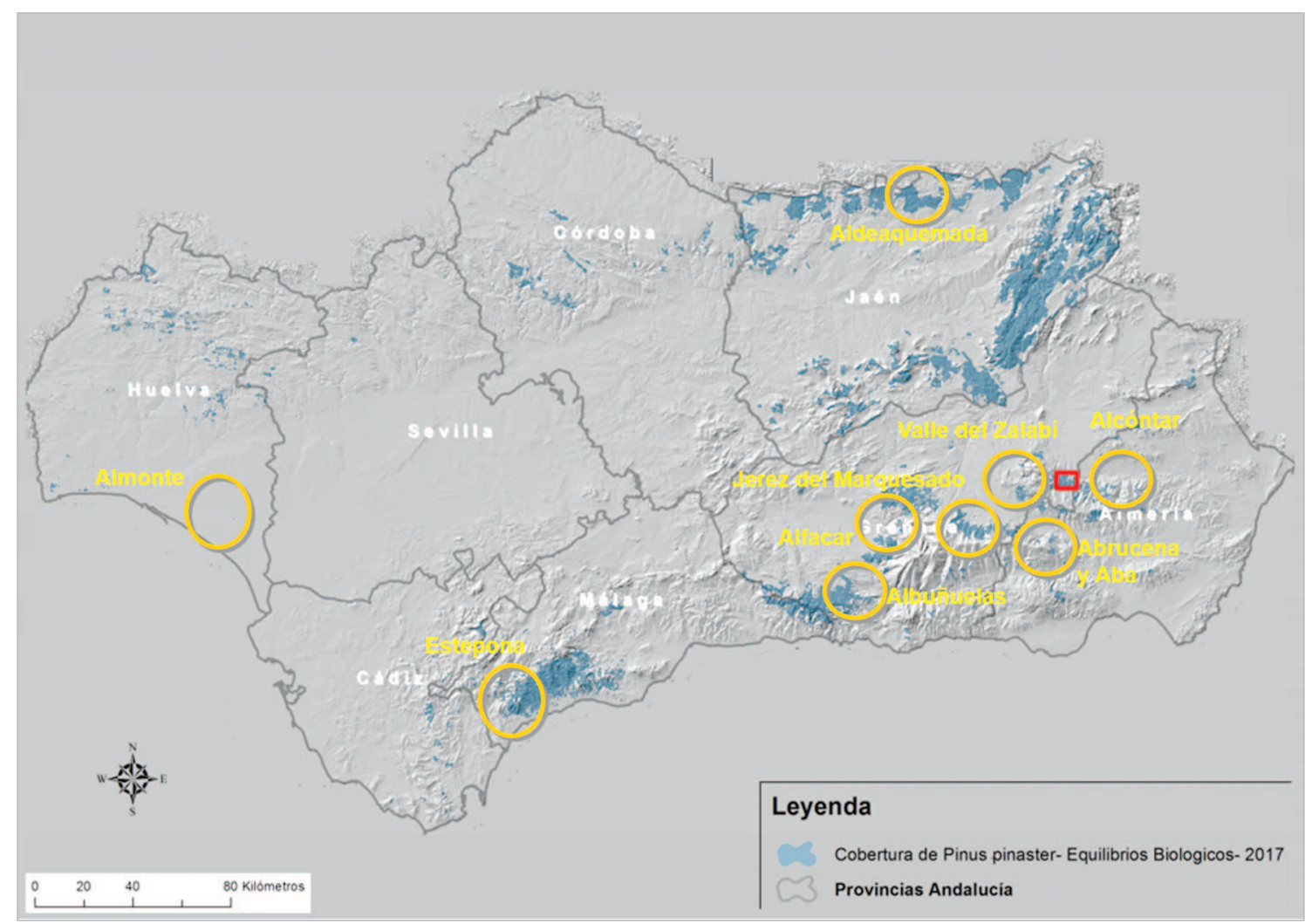

Figura 1. Situación de la zona afectada por el episodio de decaimiento en el Parque Natural de la Sierra de Baza (Granada), en verano de 2016 (en rojo) y localización de otras áreas en donde se ha muestreado la presencia de Matsucoccus feytaudi en Andalucía (en naranja).

Este episodio de decaimiento incidió sobre una parte de las repoblaciones llevadas a cabo durante la década de 1960 en el macizo serrano que sin solución de continuidad abarca la Sierra de Baza y la Sierra de los Filabres. En años anteriores ya se habían producido otros episodios severos de decaimiento sobre pinar (Navarro et al., 2007), que se han relacionado con el cambio en las condiciones climatológicas (mayor severidad de las sequías, aumento de las temperaturas) (Fernández et al., 2011; Sánchez-Salguero et al., 2012a). 


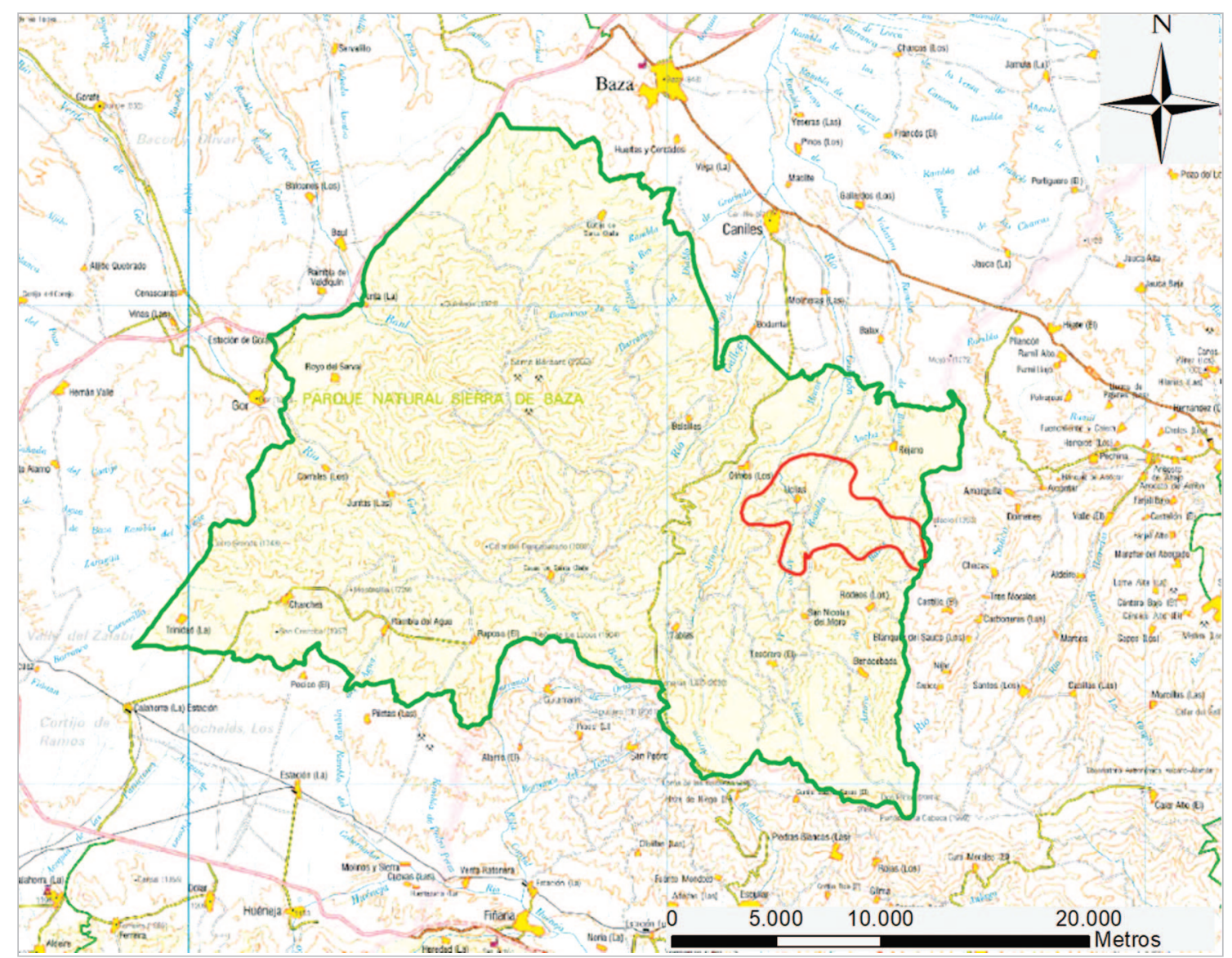

Figura 2. Mapa de detalle de la zona afectada por el episodio de decaimiento.

La muerte del arbolado de la Sierra de Baza no es un fenómeno aislado: el debilitamiento de las masas arbóreas forestales se ha constatado en diversas localidades de Andalucía, aunque se está manifestando con mayor gravedad en las repoblaciones de coníferas de la parte oriental de la Comunidad Autónoma (Sánchez-Salguero et al., 2012a; 2015). Este fenómeno del decaimiento de las repoblaciones de coníferas se inscribe en el contexto global del decaimiento forestal (Allen et al., 2010); en el ámbito mediterráneo, se ha puesto de manifiesto que se trata de fenómenos de cierta complejidad que pueden estar determinados por una multiplicidad de factores (Sánchez-Salguero et al., 2010, 2012b,c; Gómez-Aparicio et al., 2011; Navarro-Cerrillo et al., 2014).

Los episodios de decaimiento tienen una indudable componente climática. La pérdida de vigor del arbolado y las defoliaciones en ocasiones son temporales, pudiendo recuperarse los árboles si el episodio de estrés climático no es muy dilatado. Sin embargo, la respuesta favorable es mucho menor en el caso de las repoblaciones debilitadas de pinar o poco vigorosas debido a las condiciones de alta competencia por los recursos por la carencia de los tratamientos selvícolas adecuados, en particular si se comparan con formaciones naturales (Sánchez-Salguero et al., 2013; Rodríguez-Vallejo y Navarro-Cerrillo, 2019). 
El episodio del verano de 2016 presentó la particularidad de la presencia muy notoria de un artrópodo, la cochinilla corticícola Matsucoccus feytaudi Duc., 1941. cuya actuación sobre los pinos se pone de manifiesto por un descortezamiento muy característico, que adopta una forma pulverulenta. Este insecto ha sido registrado en otros eventos de debilitamiento y muerte de la especie $P$. pinaster (Cadahía y Montoya, 1967; Schvester, 1970; Jactel et al., 1998; Soria et al., 2000; Dopazo et al., 2001; Burgos et al., 2005), habiendo sido identificada como la causante de graves episodios de mortandad, como la de 120000 ha de pinos marítimos o resineros en el sur de Francia (Boutte et al., 2017).

Esta cochinilla es un insecto de pequeño tamaño (en torno a $1 \mathrm{~mm}$ de longitud) que se considera nativa en el área de distribución del pino resinero en España en donde se ha reportado su incidencia en diversas Comunidades Autónomas, habiéndose estudiado con mayor profundidad en la Comunidad Valenciana (Cadahía y Montoya, 1968; Burgos et al., 2005; Rodrigo et al., 2013).

Esta comunicación pretende aumentar el conocimiento sobre la repercusión de insectos como la cochinilla corticícola $M$. feytaudi en episodios de decaimiento forestal. El principal objetivo fue analizar la presencia del insecto durante el verano de 2016 en la Sierra de Baza (Granada), en tres zonas con distinta intensidad de afección por decaimiento. De modo secundario se determinó, con carácter comparativo, la presencia de este insecto en otras áreas de pino resinero de Andalucía.

\section{Material y Métodos}

\section{$\underline{\text { Área afectada por el decaimiento }}$}

La Sierra de Baza se encuentra situada en el Noreste de la provincia de Granada; en concreto, la zona afectada por el decaimiento pertenece a los términos municipales de Caniles y Baza. Constituye el extremo meridional de las cordilleras béticas, presentando unas características litológicas contrastadas entre las áreas pertenecientes al complejo Nevado - Filábride, de naturaleza silícea (dominado por esquistos y cuarcitas) y el complejo Alpujárride, con predominancia de materiales calizos (mármoles, calizas y dolomías). En este macizo montañoso las repoblaciones de pino resinero fueron establecidas en las décadas de los años 1960 y 1970 en altitudes intermedias (entre 800 y 1750 m.s.n.m), a una cota superior que las repoblaciones de pino carrasco (Pinus halepensis Mill.) e inferior al pino salgareño (Pinus nigra Arnold) y pino silvestre (Pinus sylvestris L.). Partiendo de una elevada densidad de plantación inicial ( $>2000$ pies ha $\left.{ }^{-1}\right)$, la implantación de los pinares sobre la gran superficie de repoblación (unas 40000 ha) resultó en general exitosa, aunque desigual en algunas zonas (principalmente en laderas soleadas de la parte oriental). Tras el crecimiento acumulado de las primeras décadas, se manifiestan en la actualidad problemas de competencia y el crecimiento ha sido afectado por la incidencia de insectos defoliadores (procesionaria del pino) y otros agentes biológicos nocivos y la tendencia hacia la reducción de las precipitaciones y el aumento de la temperatura (Fig. 3 y Fig. 4), efectos que se han podido intensificar en las esta- 
ciones con limitación edáfica o con suelos no especialmente aptos para las especies de pinos implantadas (Sánchez-Salguero et al., 2012a,b,c). La presión de los herbívoros, especialmente de la fauna cinegética, también provoca serios problemas de regeneración.

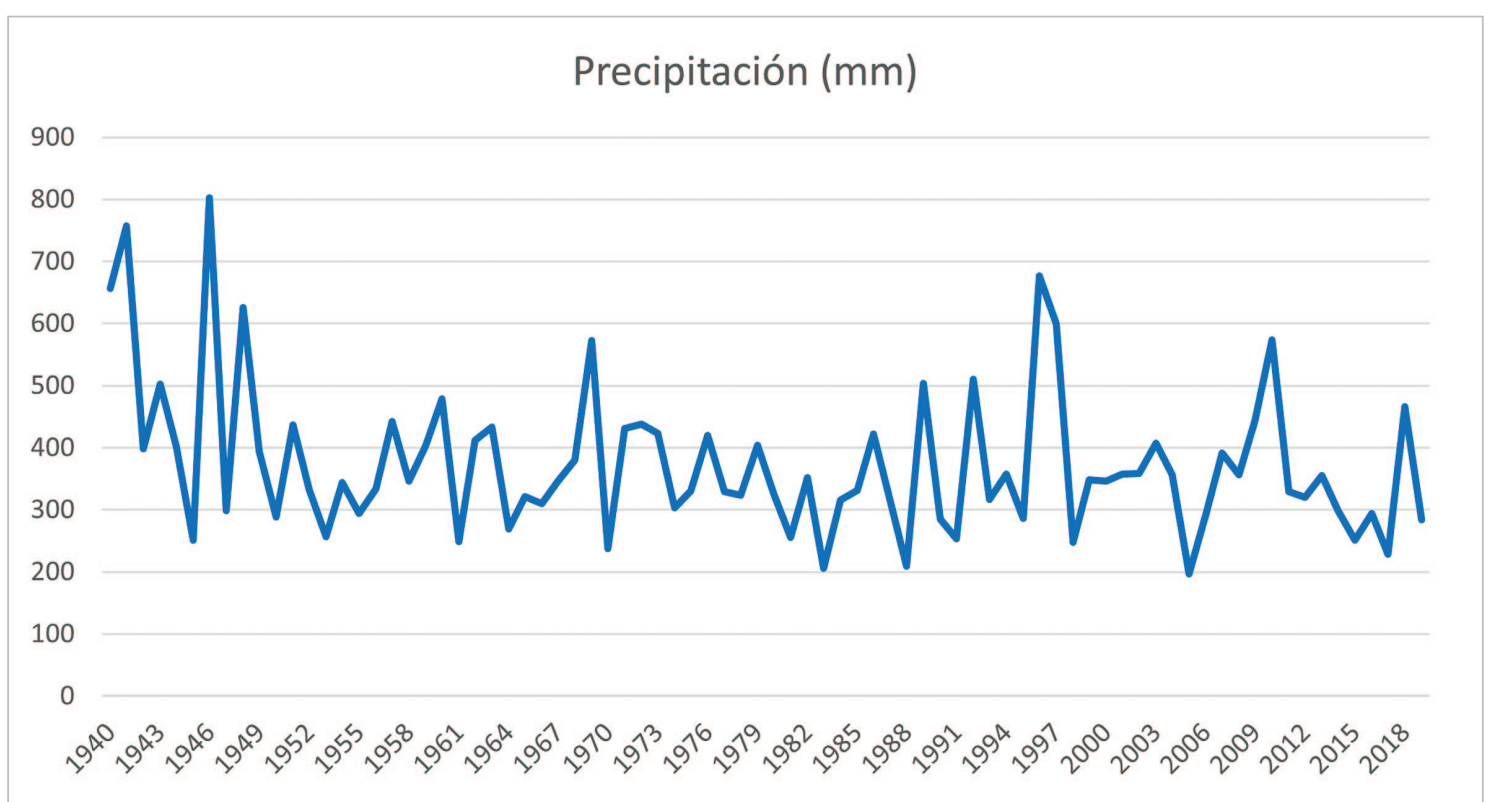

Figura 3. Precipitación anual en el Parque Natural de la Sierra de Baza (Granada), 1940-2018. Reconstrucción a partir de los datos históricos en el centroide del término municipal de Baza.

Fuente: Red de Información Ambiental de Andalucía.

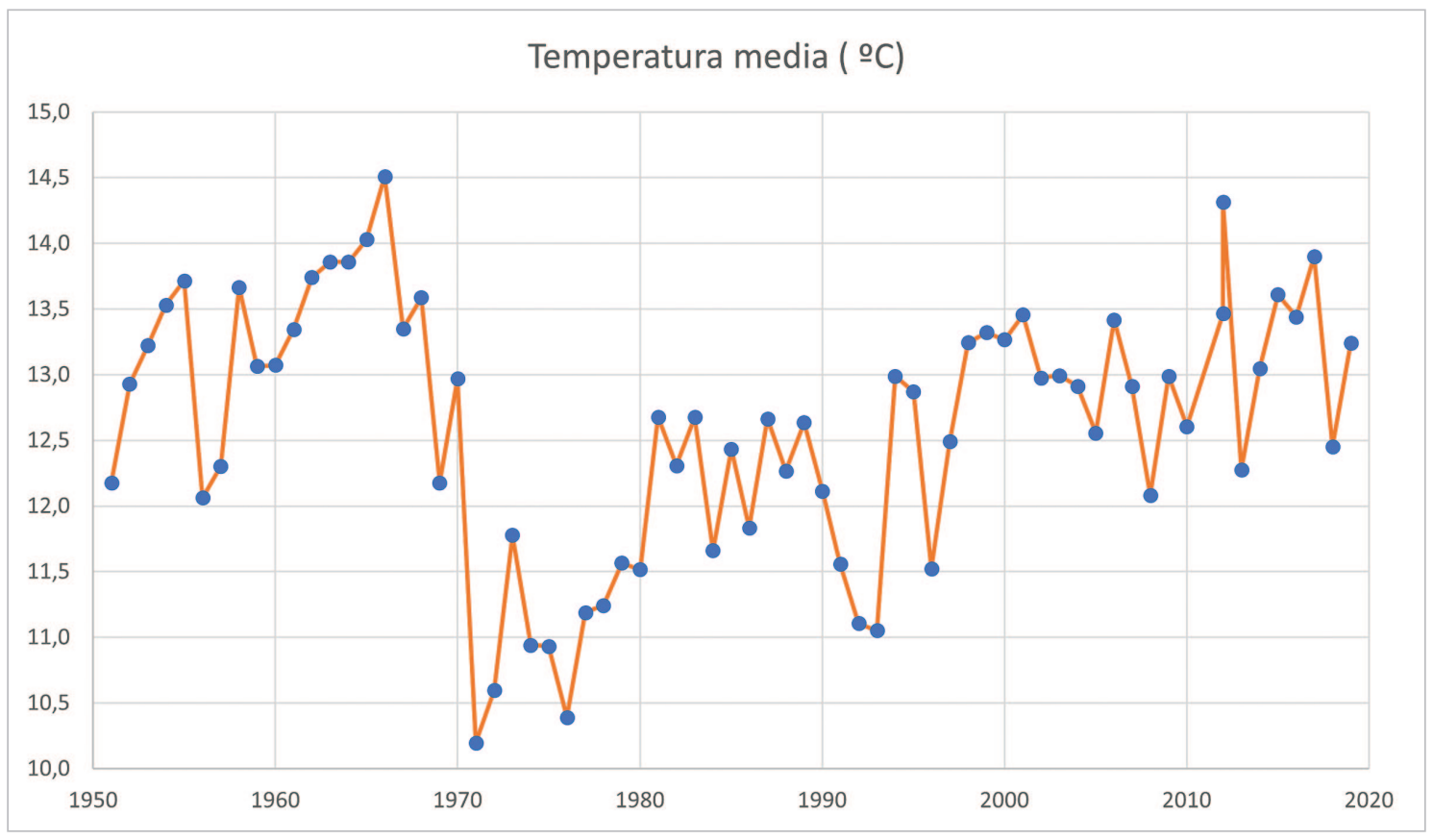

Figura 4. Temperatura media anual en el Parque Natural de la Sierra de Baza (Granada), 1940-2018. Reconstrucción a partir de los datos históricos en el centroide del término municipal de Baza. 


\section{Diseño del trampeo}

En la zona en donde se había producido con mayor intensidad durante el verano de 2016 el episodio de decaimiento de la Sierra de Baza y sus alrededores se instalaron 12 trampas adhesivas con feromona sexual para M. feytaudi proporcionadas por el INRA (Institut National de la Recherche Agronomique, Francia). Las trampas consistían en una lámina base de polipropileno blanco de 20 x $20 \mathrm{~cm}$ recubierta por un conjunto de 5 láminas de plástico transparente; estas últimas contaban con adhesivo por una de las caras. La lámina se fijaba al tronco de un pino a una altura aproximada de $1.5 \mathrm{~m}$, con una orientación preferente hacia el norte. En el centro de la placa quedaba situado el difusor de feromonas, que quedaba pinchado mediante una aguja. Cada 15 días se retiraba la lámina de plástico correspondiente, retirando previamente el difusor y volviéndolo a instalar una vez que la nueva lámina estaba dispuesta. En la segunda revisión (al cabo de un mes) se cambió el difusor de feromonas. Las trampas fueron colocadas entre el 6 y el 9 de febrero de 2017, siendo revisadas cada 15 días durante los meses de febrero, marzo y abril. En cada una de las visitas realizadas durante el periodo de muestreo se tomó una fotografía de las láminas, que fueron escaneadas posteriormente para obtener una mayor resolución de imagen. Cabe hacer constar que en los muestreos realizados en paralelo no se detectaron niveles excesivos de perforadores escolítidos.

Las trampas fueron instaladas siguiendo un muestreo sistemático estratificado por cuadrículas UTM de $1 \mathrm{~km} \times 1 \mathrm{~km}$. Para ello, previamente se delimitó sobre plano la zona afectada por el episodio de decaimiento, superponiéndole la malla de cuadrículas UTM dando como resultado un total de 48 cuadrículas, de las cuales 10 estaban situadas en su totalidad en el interior de la zona de decaimiento, 17 estaban completamente fuera de la masa afectada y 21 correspondían al borde de la zona de afección, por coincidir tanto superficie de la cuadrícula dentro del perímetro afectado como fuera de este perímetro. De las 12 trampas, 3 se ubicaron en el interior de cuadrículas correspondientes a la situación "interior", 3 en la situación "fuera de la zona afectada" y 6 en la situación "borde" (por considerar que esta ubicación podría aportar información de interés puesto que se había constatado la progersión del decaimiento por lo que en la zona borde se consideraba esperable una mayor cantidad de insectos activos. Se ubicó una única trampa por cuadrícula; dado que el número de cuadrículas era mayor al de trampas, se adoptó como criterio general de localización evitar cuadrículas contiguas. En las ubicaciones de muestreo "fuera del área afectada" y "borde", la trampa pudo situarse en árboles sin síntomas de decaimiento.

Adicionalmente, se situaron 8 trampas testigo en pinares de otras zonas de Andalucía como la Sierra de Almijara y Albuñuelas, Sierra Nevada y Jerez del Marquesado, Sierra de Baza y Valle del Zalabí (Granada); Sierra Nevada y Abla, Sierra de los Filabres y Alcóntar (Almería);,Sierra Morena y Aldeaquemada (Jaén); Los Reales de Sierra Bermeja y Estepona (Málaga); y Espacio Natural de Doñana (Huelva), tanto con síntomas de afección por decaimiento como aparentemente sanas, incluyendo áreas de pinares de repoblación y dos áreas de origen natural incluidas en dos regiones de procedencia identificadas (Alía et al., 1996) (Tab. 1 y Fig. 1). Uno 
de estos rodales (Almonte, Huelva) se encontraba aislado en el interior de una masa de pino piñonero, a gran distancia de otras zonas de pino resinero.

Posteriormente, el Servei de Gestió Forestal de Cataluña facilitó varias unidades de feromona de producción propia, que fueron colocadas en pinares de pino resinero de diversas localidades andaluzas, algunas de ellas cercanas a ubicaciones ya preestablecidas con la feromona proporcionada por el INRA, concretamente en Alfacar (La Alfaguara, Parque Natural de la Sierra de Huétor, Granada), en Jerez del Marquesado (Espacio Natural de Sierra Nevada, Granada), Aldeaquemada (Parque Natural de Despeñaperros, Jaén), y en Las Monteras (Córdoba), aunque su seguimiento fue irregular, de modo que solo se pudo constatar la efectividad del complejo feromonal.

Tabla 1. Áreas geográficas en donde se realizó la prospección y seguimiento de Matsucoccus feytaudi en Andalucía en 2017 (regiones de procedencia a partir de Alía et al., 1996).

\begin{tabular}{|c|c|c|c|c|}
\hline Localidad & $\begin{array}{c}\mathrm{N}^{\circ} \mathrm{de} \\
\text { trampas }\end{array}$ & $\begin{array}{l}\text { Unidad } \\
\text { ecológica }\end{array}$ & $\begin{array}{c}\text { Grado de } \\
\text { decaimiento }\end{array}$ & $\begin{array}{l}\text { Procedencia } \\
\text { del pinar }\end{array}$ \\
\hline $\begin{array}{l}\text { Caniles - Baza } \\
\text { (Granada) }\end{array}$ & 12 & Sierra de Baza & Grave & Repoblación \\
\hline $\begin{array}{c}\text { Abla } \\
\text { (Almería) }\end{array}$ & 1 & Sierra Nevada & Moderado & Repoblación \\
\hline $\begin{array}{l}\text { Alcóntar } \\
\text { (Almeria) }\end{array}$ & 1 & $\begin{array}{c}\text { Sierra } \\
\text { de los Filabres }\end{array}$ & Grave & Repoblación \\
\hline $\begin{array}{l}\text { Albuñuelas } \\
\text { (Granada) }\end{array}$ & 1 & $\begin{array}{c}\text { Sierra } \\
\text { de la Almijara }\end{array}$ & Leve & $\begin{array}{c}\text { Natural (Región procedencia } 19 . \\
\text { Almijara-Nevada) }\end{array}$ \\
\hline $\begin{array}{l}\text { Valle del Zalabí } \\
\text { (Granada) }\end{array}$ & 1 & Sierra de Baza & Leve & Repoblación \\
\hline $\begin{array}{l}\text { Jerez del Marquesado } \\
\text { (Granada) }\end{array}$ & 1 & Sierra Nevada & Moderado & Repoblación \\
\hline $\begin{array}{l}\text { Estepona } \\
\text { (Málaga) }\end{array}$ & 1 & $\begin{array}{l}\text { Los Reales de } \\
\text { Sierra Bermeja }\end{array}$ & Sin síntomas & $\begin{array}{c}\text { Natural(Región procedencia } 20 . \\
\text { Sierra Bermeja) }\end{array}$ \\
\hline $\begin{array}{l}\text { Aldeaquemada } \\
\text { (Jaén) }\end{array}$ & 1 & Sierra Morena & Leve & Repoblación \\
\hline $\begin{array}{l}\text { Almonte } \\
\text { (Huelva) }\end{array}$ & 1 & Doñana & Sin síntomas & Repoblación \\
\hline
\end{tabular}

\section{Tratamiento de la información y análisis estadístico}

Tras una fase de entrenamiento para la identificación en las imágenes de los ejemplares de machos de M. feytaudi capturados en la placa de la trampa, se probó a llevar a cabo el recuento de especímenes mediante el software ImageJ (https://imagej.nih.gov/ij/); pero debido al alto grado de superposición de ejemplares, la capacidad discriminatoria no se consideró apropiada, por lo cual la contabilización final se hizo de visu. La contabilización se pudo realizar de manera exhaustiva en la ma- 
yor parte de los casos, excepto cuando el gran recubrimiento de la base por ejemplares de $M$. feytaudi impidió la separación visual entre ejemplares, asignando en estos casos una cifra indicativa (más de 5000 ejemplares) basada en estimaciones más fiables que sí se pudieron hacer en otras placas (Fig. 5 y Fig. 6).

Los datos correspondientes al seguimiento en la Sierra de Baza (12 trampas en 3 ubicaciones diferenciadas: "interior", "fuera" y "borde") fueron analizados mediante un test de comparación de medias (ANOVA Tuckey HSD post hoc $p<0.05$ ) para los diferentes momentos temporales de muestreo. El resto de localidades ( $8 \mathrm{em}$ plazamientos adicionales con una trampa en cada uno de ellos) aportaron información de referencia para realizar una evaluación preliminar de la presencia de $M$. feytaudi a escala regional, contrastándose los valores numéricos de captura con el grado de incidencia del decaimiento.

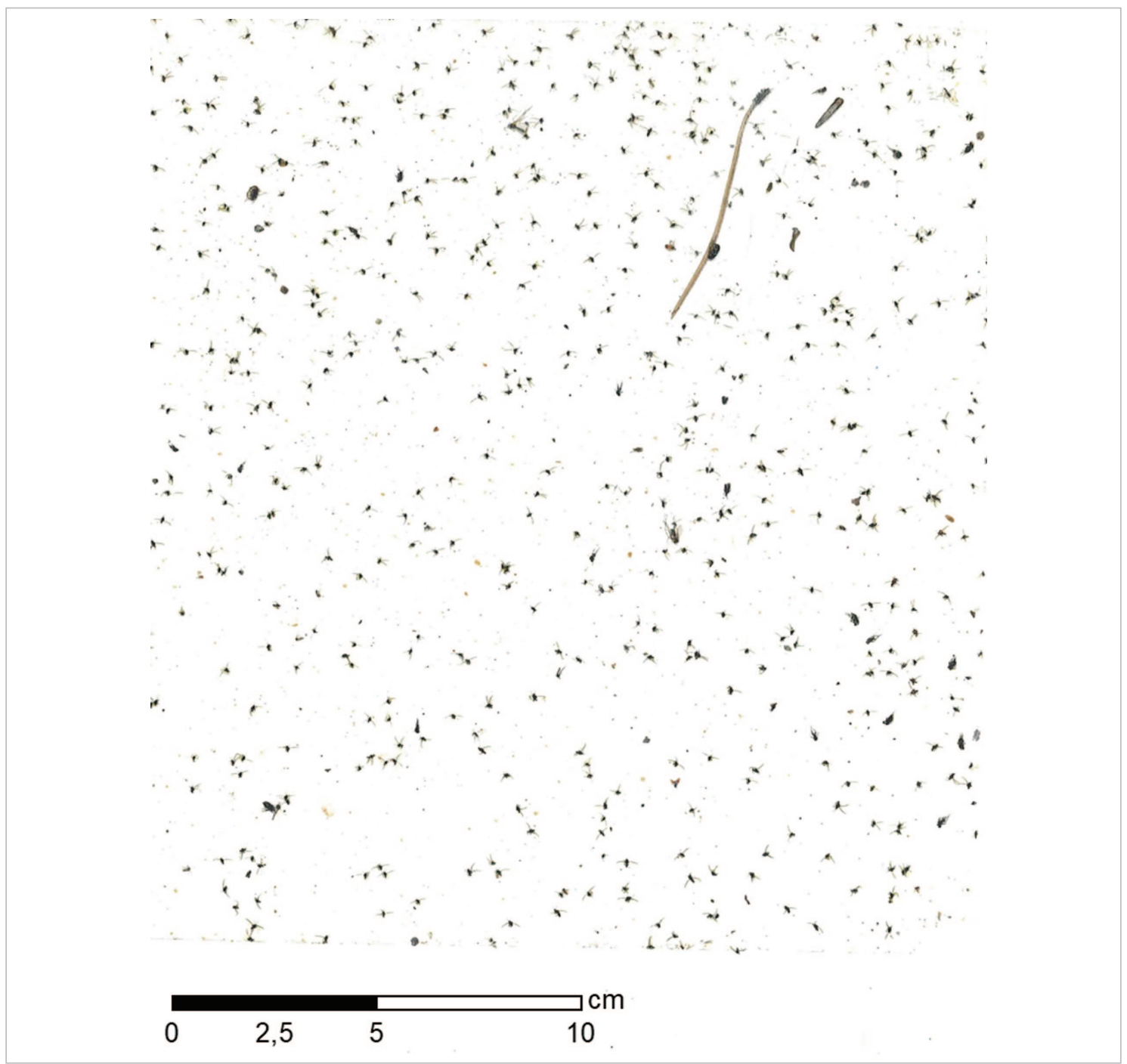

Figura 5. Imagen de trampa con capturas de Matsucoccus feytaudi correspondientes a la estación T-20 de la Sierra de Baza (Granada). 


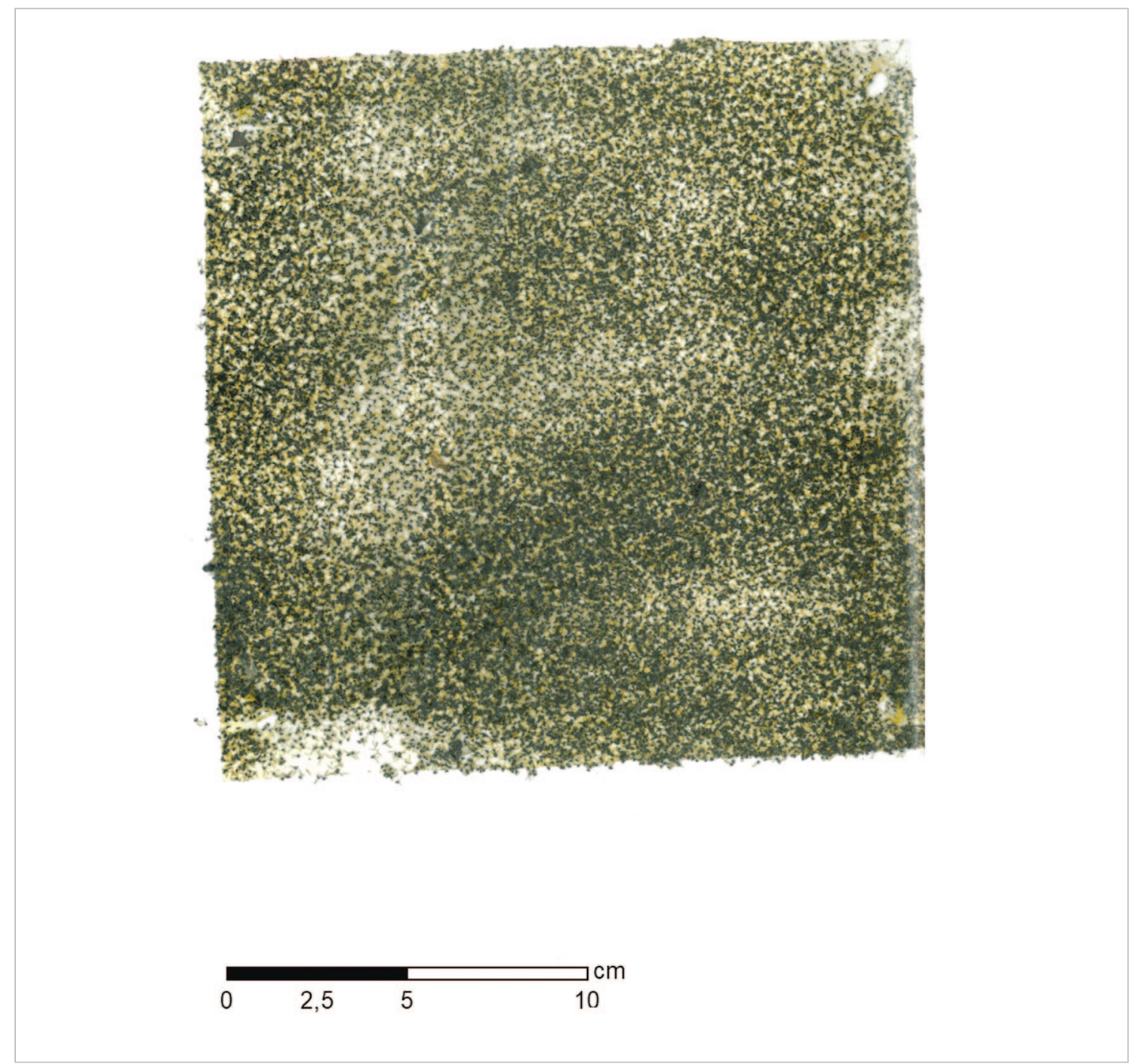

Figura 6. Imagen de trampa con capturas de Matsucoccus feytaudi correspondientes a la estación de Aldeaquemada (Jaén).

\section{Resultados}

\section{Presencia de Matsucoccus feytaudi en la Sierra de Baza}

Las capturas fueron superiores en las localizaciones "dentro" y "borde" del área afectada por el proceso de decaimiento de la Sierra de Baza (Tab.2, Fig. 7). Las diferencias entre dentro, fuera y borde no muestran diferencias significativas (Tab. 2). Es de resaltar que en las dos últimas fechas de muestreo los valores de captura se igualaron en las tres localizaciones.

El número de ejemplares totales capturados por estación ha variado entre $124 \mathrm{y}$ 4082. La presencia de $M$. feytaudi ha sido más cuantiosa en las trampas situadas en la parte meridional de la zona afectada (trampas $\left.n^{\circ} 26,32,35\right)$, mientras que el 
menor número de ejemplares se han contabilizado en la parte septentrional (trampas $\mathrm{n}^{\mathrm{o}} 2,5,11$ ), distribución coincidente con la progresión del decaimiento que ha mostrado una dirección de evolución sur a norte.

Las curvas de evolución de capturas de machos no son sincrónicas en todas las localizaciones de las trampas (Fig. 7), aunque se puede concluir que los máximos se produjeron de forma general entre el 7 y el 22 de marzo.

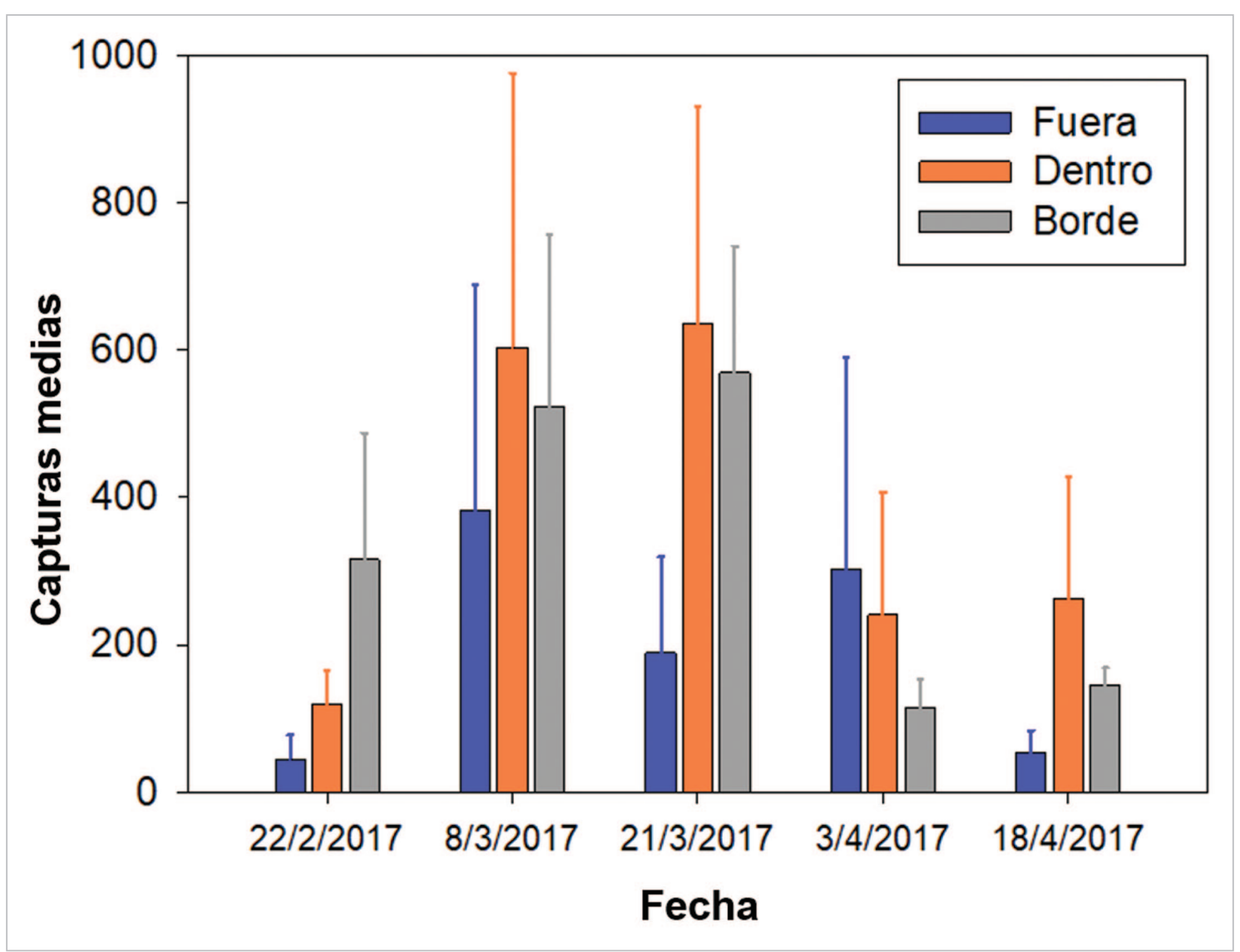

Figura 7. Evolución de capturas de machos de Matsucoccus feytaudi en las trampas de muestreo con feromonas en las diferentes localizaciones de la Sierra de Baza (Granada). Se muestra la media y el error estándar.

\section{Presencia de Matsucoccus feytaudi en otras áreas de pinar resinero en Andalucía}

En todas las zonas en donde se colocaron trampas de muestreo se obtuvieron registros de $M$. feytaudi, excepto en la localidad de Almonte, presentando variaciones numéricas muy notables (Fig. 8 y Tab. 3). 
Tabla 2. Capturas de ejemplares de Matsucoccus feytaudi en las trampas de muestreo con feromona sexual situadas en el Parque Natural de la Sierra de Baza (Granada), en tres ubicaciones respecto a la afección por decaimiento.

\begin{tabular}{|c|c|c|c|c|c|c|}
\hline $\begin{array}{l}\text { Ubicación/ } \\
\text { trampa }\end{array}$ & $\begin{array}{c}21 \text { a } 22 / \\
2 / 2017\end{array}$ & $\begin{array}{c}7 \text { a 9/ } \\
3 / 2017\end{array}$ & $\begin{array}{c}21 \text { a } 24 / \\
3 / 2017\end{array}$ & $\begin{array}{c}3 \text { a } 7 / \\
7 / 2017\end{array}$ & $\begin{array}{c}18 \text { a } 21 / \\
4 / 2017\end{array}$ & TOTAL \\
\hline \multicolumn{7}{|l|}{ Fuera } \\
\hline $\mathbf{T} 2$ & 9 & 101 & 89 & 22 & 28 & 249 \\
\hline T11 & 13 & 49 & 30 & 10 & 22 & 124 \\
\hline T35 & 112 & 996 & 448 & 878 & 113 & 2547 \\
\hline Media & 44.67 & 382.00 & 189.00 & 303.33 & 54.33 & \\
\hline Desv. est. & 58.35 & 532.37 & 226.23 & 497.71 & 50.90 & \\
\hline \multicolumn{7}{|l|}{ Dentro } \\
\hline T14 & 128 & 235 & 401 & 102 & 135 & 1001 \\
\hline T17 & 192 & 1.348 & 1.223 & 572 & 591 & 3926 \\
\hline T23 & 39 & 227 & 284 & 49 & 65 & 664 \\
\hline Media & 119.67 & 603.33 & 636.00 & 241.00 & 263.67 & \\
\hline Desv. est. & 76.84 & 644.91 & 511.71 & 287.88 & 285.63 & \\
\hline \multicolumn{7}{|l|}{ Borde } \\
\hline T5 & 13 & 33 & 47 & 40 & 48 & 181 \\
\hline T8 & 17 & 133 & 149 & 75 & 148 & 522 \\
\hline T20 & 132 & 489 & 686 & 83 & 207 & 1597 \\
\hline T26 & 1089 & 1465 & 1094 & 246 & 188 & 4082 \\
\hline T29 & 137 & 72 & 502 & 26 & 120 & 857 \\
\hline T32 & 509 & 941 & 940 & 223 & 164 & 2777 \\
\hline Media & 316.17 & 522.17 & 569.67 & 115.50 & 145.83 & \\
\hline Desv. est. & 419.97 & 575.99 & 419.60 & 94.86 & 56.73 & \\
\hline \multicolumn{7}{|l|}{ ANOVA } \\
\hline $\mathrm{F}$ & 0.863 & 0.112 & 1.117 & 0.506 & 1.616 & \\
\hline $\mathrm{p}$ & 0.454 & 0.895 & 0.369 & 0.619 & 0.251 & \\
\hline
\end{tabular}

El número total de capturas ha oscilado entre 419 machos en Albuñuelas (decaimiento leve) hasta decena de miles en el caso de Aldeaquemada (decaimiento leve), con $n=539$ en Alcóntar (decaimiento grave), $n=617$ en Abla (moderado), $n=1586$ en Valle del Zalabí (leve), $\mathrm{n}=2202$ en Estepona ( $\operatorname{cin}$ síntomas) y $\mathrm{n}=3146$ en Jerez del Marquesado (moderado). En Almonte (sin síntomas) no ha habido ninguna captura,

Las curvas de evolución del número de ejemplares para cada localidad presentan diferentes momentos de máximo poblacional: el 24/2 en Aldeaquemada; el 10/3 en Albuñuelas y Jerez del Marquesado; el 21/3 en Abla y Valle del Zalabí; y el 19/4 en Estepona y Alcóntar. 
Tabla 3. Capturas de ejemplares de Matsucoccus feytaudi en las trampas de muestreo con feromona sexual situadas en otras localidades de Andalucía (8 localizaciones).

\begin{tabular}{|c|c|c|c|c|c|c|}
\hline & $\begin{array}{l}22 \text { a } 24 / \\
2 / 2017\end{array}$ & $\begin{array}{l}10 / 3 / \\
2017\end{array}$ & $\begin{array}{l}21 \text { a } 22 / \\
3 / 2017 \\
\end{array}$ & $\begin{array}{r}7 / 4 / \\
2017\end{array}$ & $\begin{array}{l}19 \text { a } 20 / \\
4 / 2017\end{array}$ & $\begin{array}{l}2 / 5 / \\
2017\end{array}$ \\
\hline $\begin{array}{l}\text { Abla } \\
\text { (Sierra Nevada) }\end{array}$ & 61 & 133 & 235 & 78 & 66 & 44 \\
\hline $\begin{array}{l}\text { Alcóntar } \\
\text { (Sierra de los Filabres) }\end{array}$ & 19 & 77 & 157 & 98 & 188 & $\begin{array}{c}\text { no } \\
\text { muestreado }\end{array}$ \\
\hline $\begin{array}{l}\text { Estepona } \\
\text { (Los Reales, Sierra Bermeja) }\end{array}$ & 122 & 151 & 289 & 425 & 905 & 310 \\
\hline $\begin{array}{l}\text { Aldeaquemada } \\
\text { (Sierra Morena) }\end{array}$ & $>5000$ & $>5000$ & 1.00 & 600 & $\begin{array}{c}\text { no } \\
\text { muestreado }\end{array}$ & $\begin{array}{c}\text { no } \\
\text { muestreado }\end{array}$ \\
\hline $\begin{array}{l}\text { Albuñuelas } \\
\text { (Sierra de la Almijara) }\end{array}$ & 75 & 189 & 97 & 58 & $\begin{array}{c}\text { no } \\
\text { muestreado }\end{array}$ & $\begin{array}{c}\text { no } \\
\text { muestreado }\end{array}$ \\
\hline $\begin{array}{l}\text { Valle del Zalabí } \\
\text { (Sierra de Baza) }\end{array}$ & 31 & 360 & 1015 & 112 & 68 & $\begin{array}{c}\text { no } \\
\text { muestreado }\end{array}$ \\
\hline $\begin{array}{l}\text { Jerez del Marquesado } \\
\text { (Sierra Nevada) }\end{array}$ & 1211 & 1468 & 276 & 191 & $\begin{array}{c}\text { no } \\
\text { muestreado }\end{array}$ & $\begin{array}{c}\text { no } \\
\text { muestreado }\end{array}$ \\
\hline $\begin{array}{l}\text { Almonte } \\
\text { (P. N. Doñana) }\end{array}$ & 0 & 0 & 0 & 0 & $\begin{array}{c}\text { no } \\
\text { muestreado }\end{array}$ & $\begin{array}{c}\text { no } \\
\text { muestreado }\end{array}$ \\
\hline
\end{tabular}

\section{Otras especies de artrópodos capturadas en el trampeo}

De forma complementaria, se han identificado en las trampas las siguientes especies de artrópodos depredadores (véase también Gómez de Dios et al., 2017; García-Sánchez et al., 2019): los coccinélidos Iberorhyzobius rondensis (Eizaguirre, 2004) (véase Tavares et al., 2015) y Myrrha octodecimguttata ssp. formosa (Costa, 1849), el antocórido Elatophilus nigricornis (Zetterstedt, 1838) (véase Fabre et al., 2000), la mosca serpiente Subilla aliena (Navás, 1915), el melírido Falsomelyris granulata (Fabricius, 1792), dasítidos del género Aplocnemus (Stephens, 1830) (véase Branco et al., 2011), carábidos del género Dromius (Bonelli, 1810), o varios estafilínidos sin identificar, alguno de ellos perteneciente al género Quedius (Stephens, 1829).

Paralelamente, se observaron otras especies que podrían estar asociadas al proceso de decaimiento, especialmente el anóbido Ernobius cf. mollis (Linnaeus, 1758), que ha sido detectado de forma muy abundante también en la corteza de pinos resineros con presencia de la cochinilla; bupréstidos de los géneros Anthaxia (Eschscholtz, 1829), Phaenops (Dejean, 1833) y Buprestis (Linnaeus, 1758); hemípteros fulguromorfos de la familia Cixidae y la hormiga Crematogaster auberti (Emery, 1869), que también se ha observado transportando hembras de M. feytau$d i$, aunque se desconoce si es manifestación de una asociación mutualista o si existe depredación sobre la cochinilla. 


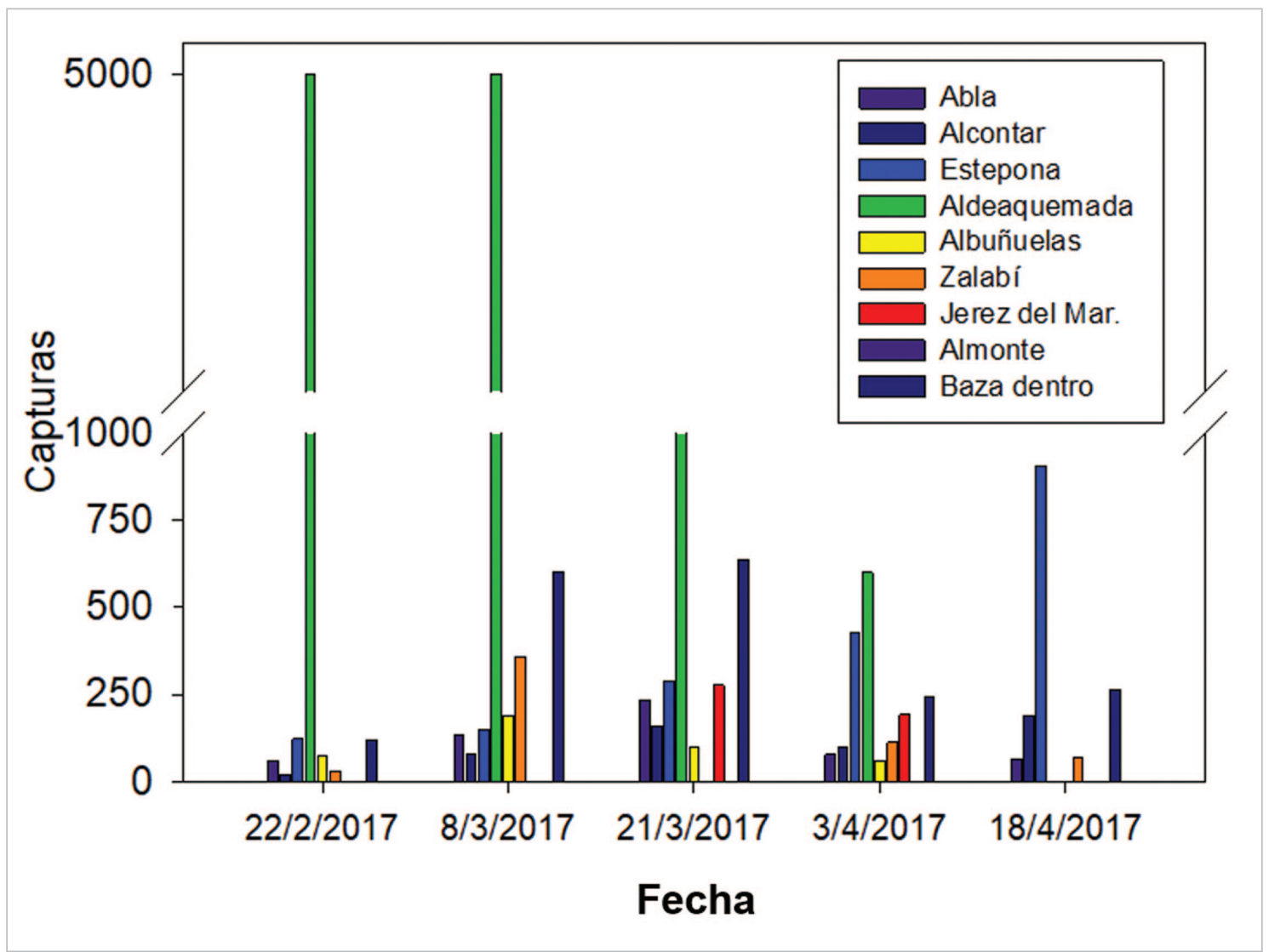

Figura 8. Evolución de capturas totales de machos de Matsucoccus feytaudi en las trampas de muestreo con feromonas en las diferentes localizaciones estudiadas y comparación con las capturas medias en las localizaciones Baza-dentro (ver Tab. 3).

Igualmente se han encontrado numerosos taxones adicionales en las trampas adhesivas: diversos artrópodos que habitan este tipo de formaciones forestales y que también han sido observados en la zona cortical de pinos resineros afectados, probablemente de carácter saprófito, como algunos derméstidos del género Attagenus ssp (Latreille, 1802) y algunas especies de microlepidópteros sin determinar.

\section{4.- Discusión}

Se ha constatado la presencia de la cochinilla corticícola $M$. feytaudi en la mayor parte de las masas forestales de $P$. pinaster prospectadas en Andalucía, tanto las de origen natural como las procedentes de repoblación (Fig. 8). La excepción es el rodal aislado de Almonte, cuya explicación podría consistir precisamente en el aislamiento en relación con otros pinares de pino resinero y situarse en una matriz compuesta por pinares de pino piñonero.

Los datos obtenidos en cuanto al periodo de vuelo de los machos de M. feytau$d i$, con presencia de ejemplares durante todo el lapso temporal en el que se realizó 
el seguimiento (desde febrero hasta mayo), y presentando máximos que parecen situarse en el mes de marzo (aunque con la existencia de diferencias locales), son coherentes con los aportados por otros trabajos que han abordado la fenología de la especie (Cadahía y Montoya, 1967; Dopazo et al., 2001; Burgos et al., 2005). A1 haberse interrumpido el muestreo en mayo de 2017 no se ha podido comprobar si los machos vuelan de forma continua en un período de tiempo más amplio, tal y como indican Branco et al. (2011) en Portugal.

Igualmente es reseñable la constatación por parte del equipo de campo de la presencia de $M$. feytaudi en otros ámbitos geográficos de Andalucía, aunque ésta no se ha cuantificado, como en los pinares de Sierra Morena de Córdoba (monte "Las Monteras", Villaviciosa de Córdoba), del Parque Natural de la Sierra de Huétor (Alfacar, Granada) y de Oria (Almería).

En algunos casos el número de ejemplares capturados en las trampas ha tenido un orden de magnitud de miles de ejemplares por placa, recubriéndolas en su totalidad, cifras que superan los registros de estudios anteriores (Burgos et al., 2005; Rodrigo et al., 2013).

Como se ha puesto de manifiesto, es notable la presencia de M. feytaudi en los pinares muestreados de pino negral de Andalucía, tanto naturales como de repoblación, incluso en los que no mostraban un debilitamiento severo (es interesante reseñar el caso de Los Reales de Sierra Bermeja, pinar de carácter natural sin síntomas de decaimiento). De hecho, los pinares de la Sierra de Baza aledaños a la zona en donde se produjo el episodio de decaimiento del verano anterior (julio y agosto de 2016) no han presentado los valores más elevados de capturas registrados en este estudio, habiendo sido superados en número de machos contabilizados por la localidad de Aldeaquemada (Jaén) en donde la incidencia de síntomas visibles de decaimiento ha sido menor (no obstante, se ha de tener en cuenta que los muestreos en la Sierra de Baza fueron hechos en la campaña posterior al episodio de muerte del arbolado, no disponiéndose de información previa sobre la población de M. feytau$d i$ en la zona afectada). En este sentido, se ha planteado que pueda existir una mayor resistencia de algunas procedencias de pinos resineros en base a una coevolución entre $P$. pinaster y $M$. feytaudi que se habría plasmado en diferenciaciones genéticas entre ecotipos (Schvester y Ughetto, 1986; Burban et al., 1999).

Igualmente es reseñable la presencia de fauna depredadora que pueda estar actuando como controladora de desequilibrios poblaciones de $M$. feytaudi como la cochinilla E. nigricornis, registrada por Burgos et al. (2005) en la Comunidad Valenciana, y el coccinélido I. rondensis, estudiado por Tavares et al. (2015), además de taxones adicionales no registrados con anterioridad en la bibliografía.

El episodio de decaimiento de $P$. pinaster en la Sierra de Baza se ha correspondido con elevadas poblaciones de $M$. feytaudi; sin embargo, a la vista de los cuantiosos niveles poblacionales encontrados en zonas de pinar sin decaimiento o con una menor incidencia no es posible concluir que este insecto constituya el agente primario del decaimiento, tal y como ha sido identificado en otras zonas (Boutte et al., 2017) o se ha propuesto como posibilidad (Dopazo et al., 2011). Lo anterior nos lleva a pensar que esta cochinilla pudiera estar actuando como un agente secundario o factor de contribución siendo favorecida su explotación demográfica por el pro- 
pio debilitamiento del pinar, acelerando significativamente el desenlace final de mortalidad (sensu Manion, 1981).

\section{Conclusiones}

Se ha constatado una elevada presencia de la cochinilla del pino en los pinares prospectados, tanto en la zona muy afectada por el decaimiento de la Sierra de Baza del verano de 2016 como en otros pinares de pino negral de Andalucía. Los niveles de presencia pueden ser considerados en algunos casos como una explosión poblacional, atendiendo a las cifras previas aportadas por la bibliografía. Las curvas de vuelo de la cochinilla muestran una fenología específica en cada área geográfica.

En relación con la fauna auxiliar, se ha constatado la presencia de depredadores de $M$. feytaudi como I. rondensis y E. nigricornis, junto con otros taxones de posible carácter depredador no señalados en la bibliografía.

Los elevados niveles poblaciones de Matsucoccus registrados en pinares sin síntomas severos de decaimiento inducen a considerar que la cochinilla podría haberse comportado como un agente secundario en el proceso de decaimiento, acelerando la muerte del arbolado debilitado por las condiciones climáticas actuales, las características edáficas, la competencia de la masa forestal, incluso contemplando la posibilidad de que el origen genético de las plantas utilizadas en la repoblación hubiera sido poco adecuado para la estación. A este respecto, sería oportuno continuar profundizando en el conocimiento de la distribución territorial y la biología y ecología de Matsococcus feytaudi y, en especial, los factores ambientales que inducen situaciones de explosión demográfica como la acaecida en la Sierra de Baza en 2016.

\section{Agradecimientos}

A Hervé Jactel, Carole Kerdelhué e Inge Van Halder, del Institute National de la Recherche Agronomique (INRA), Francia, por el asesoramiento en el trampeo y el abastecimiento de feromonas y otro material de trampeo. A Jorge Heras de la Secció de Planificació, Servei de Gestió Forestal Generalitat de Catalunya, por haber apoyado el seguimiento a través del abastecimiento de feromona complementaria. A Gloria Cabezas, Rosario Lozano y Francisco García, Agencia de Medio Ambiente y Agua (CMAYOT) por el apoyo en el trabajo de campo. A Manuel Baena por la confirmación de la identidad de Elatophilus nigricornis y a Kiko Gómez Abad por la de Crematogaster auberti. A Raúl Sánchez Salguero por su apoyo en la revisión final del trabajo. 


\section{Bibliografía}

Alía Miranda, R.; Martín Albertos, S.; De Miguel y Del Ángel, J.; Galera Peral, R.M.; Agúndez Leal, D.; Gordo Alonso, J.; Salvador Nemoz, L.; Catalán Bachiller, G.; Gil Sánchez, L.A.; 1996. Las regiones de procedencia de Pinus pinaster Aiton. Dirección General de Conservación de la Naturaleza, Madrid.

Allen, C.D.; Macalady, A.K.; Chenchouni, H.; Bachelet, D.; McDowell, N.; Vennetier, M.; Kitzberger, T.; Rigling, A.; Breshears, D.D.; Hogg, E.H.; Gonzalez, P.; Fensham, R.; Zhang, Z.; Castro, J.; Demidova, N.; Lim, J.H.; Allard, G.; Running, S.W.; Semercis, A.; Cobb, N.; 2010. A global overview of drought and heat-induced tree mortality reveals emerging climate change risks for forests. For. Ecol. Manag. 259, 660-684. https://doi.org/10.1016/j.foreco.2009.09.001

Boutte, B.; Daubree, J.B.; Goudet, M.; 2017. Prévention et lutte contre la cochenille du tronc du pin maritime. Synthèse des travaux de la recherche et des observations du Département de la Santé des Forêts. Ministére de l'Agriculture et de l'Alimentation.

Branco, M.; van Halder, I.; Franco, J.C.; Constantin, R.; Jactel, H.; 2011. Prey sex pheromone as kairomone for a new group of predators (Coleoptera: Dasytidae, Aplocnemus spp.) of pine bast scales. Bull. Entomol. Res. 101(6), 1-8. https://doi.org/10.1017/S0007485310000696

Burban, C.H.; Petit, R.J.; Carcreff, E.; Jactel, H.; 1999. Rangewide variation of the maritime pine bast scale Matsococcus feytaudi Duc. (Homoptera: Matsucoccidae) in relation to genetic structure of its host. Mol. Ecol. 8, 1593-1602. https://doi.org/10.1046/j.1365-294x. 1999.00739.x

Burgos, G.; Rodrigo, M.E.; Pérez-Laorga, E.; Sabater, M.; 2005. Prospección y estudio de Matsucoccus feytaudi Duc. y sus enemigos naturales en la Comunidad Valenciana. IV Congreso Forestal Nacional, Zaragoza.

Cadahía, D.; Montoya, R.; 1967. Matsucoccus feytaudi Duc., un peligroso enemigo para Pinus pinaster Sol. en España. Boletín del Servicio de Plagas 20, 95-101.

Cadahía, D., Montoya, R., 1968. Prospección de Matsucoccus feytaudi Duc., y Matuscoccus pini Green (Homóptera Margarodidae) en la mitad norte de España. Boletín del Servicio de Plagas 22, 113-134.

Dopazo, C., Pérez-Laorga, E., Giménez, A., 2001. Presencia de la cochinilla del pino rodeno Matsucoccus feytaudi Duc. en la Comunidad Valenciana. III Congreso Forestal Español, Granada.

Fabre, J.P.; Menassieu, P.; Foing, J.J.; Chalon, A.; 2000. Biology and ecology of Elatophilus nigricornis Zetterstedt (Hemiptera Anthocoridae) predator of Matsucoccus feytaudi Ducasse (Homoptera Matsucoccidae) in the South-East of France. Ann. For. Sci. 57(8), 777-792. https://doi.org/10.1051/forest:2000104

Fernández Cancio, A.; Navarro Cerrillo, R.M.; Sánchez Salguero, R.; Fernández Fernández, R.; Manrique Menéndez, E.; 2011. Viabilidad fitoclimática de las repoblaciones de pino silvestre (Pinus sylvestris L.) en la Sierra de los Filabres (Almería). Ecosistemas 20, 124-144.

García-Sánchez, P.; Barranco-Vega, P.; Gómez de Dios, M.A.; 2019. Incidencia de artrópodos en trampas de feromonas para Monochamus galloprovincialis asociada al decaimiento de Pinus pinaster en la Sierra de Baza. IV Congreso de Biodiversidad y Conservación de la Naturaleza. Puerto Real, Cádiz, p 36. https://conserbio.files.wordpress.com/2019/09/libro_ resumenes_iv_conserbio.pdf

Gómez-Aparicio, L.; García-Valdés, R.; Ruiz-Benito, P.; Zavala, M.A.; 2011. Disentangling the 
relative importance of climate, size and competition on tree growth in Iberian forests: implications for management under global change. Glob. Change Biol. 17, 2400-2414. https: //doi.org/10.1111/j.1365-2486.2011.02421.x

Gómez de Dios, M.A.; Muñoz Risueño, A.; Alguacil Picón, F.; Guzmán Álvarez, J.R.; Nebot Sanz, B.; Cobos Aguirre, J.; Carrasco Gotarredona, A.; Rodríguez Reviriego, S.; 2017. Entomofauna asociada al decaimiento de Pinus pinaster Aiton en Andalucía. El caso particular de Matsucoccus feytaudi Ducasse, 1941. In: XXXIII Jornadas de la Asociación Española de Entomología, Almería.

Jactel, H.; Menassieu, P.; Ceria, A.; Burban, C.; Regad, J.; Normand, S.; Carcreff, E.; 1998. Une pullulation de la cochenille Matsucoccus feytaudi provoque un début de dépérissement du pin maritime en Corse. Rev. For. Francaise 1, 33-45. https://doi.org/10.4267/2042/5510

Manion, P.D.; 1981. Tree Disease Concepts. Prentice Hall, Upper Saddle River (NJ), pp. 409.

Navarro Cerrillo, R.M.; Varo, M.A.; Lanjeri, S.; Hernández-Clemente, R.; 2007. Cartografía de defoliación en los pinares de pino silvestre (Pinus sylvestris L.) y pino salgareño (Pinus nigra Arnold.) en la Sierra de los Filabres. Ecosistemas 16, 163-171.

Navarro-Cerrillo, R.M.; Sánchez-Salguero, R.; Manzanedo, R.D.; Camarero, J.J.; FernándezCancio, A.; 2014. Site and age condition the growth responses to climate and drought of relict Pinus nigra subsp. salzmannii populations in Southern Spain. Tree Ring Res. 70, $145-$ 155. https://doi.org/10.3959/1536-1098-70.2.145

Rodrigo, E.; Catalá-Oltra, M.; Pérez-Laorga, E.; Baena, M.; 2013. Monitoring of Matsucoccus feytaudi and its natural enemies in Spain using sticky tapes and pheromone traps. Eur. J. Entomol 110, 301-310. https://doi.org/10.14411/eje.2013.043

Rodríguez-Vallejo, C.; Navarro-Cerrillo, R.M.; 2019. Contrasting response to drought and climate of planted and natural Pinus pinaster Aiton forests in Southern Spain. Forest 10, 603. https://doi.org/10.3390/f10070603

Sánchez-Salguero, R.; Navarro-Cerrillo, R.M.; Camarero, J.J.; Fernández-Cancio, A.; 2010. Drought-induced growth decline of Aleppo and maritime pine forests in South-eastern Spain. For Syst 19, 458-469. https://doi.org/10.5424/fs/2010193-9131

Sánchez Salguero, R.; Navarro-Cerrillo, R.M.; Camarero, J.L.; Fernández-Cancio, A.; Swetnam, T.W.; Zavala, M.A.; 2012a. Vulnerabilidad frente a la sequía de repoblaciones de dos especies de pinos en su límite meridional en Europa. Ecosistemas 21, 31-40. https://doi.org /10.7818/ECOS.2012.21-3.05

Sánchez-Salguero, R.; Navarro-Cerrillo, R.M.; Camarero, J.J.; Fernández-Cancio, A.; 2012b. Selective drought-induced decline of pine species in Southeastern Spain. Clim. Change 113, 767-785. https://doi.org/10.1007/s10584-011-0372-6

Sánchez-Salguero, R.; Navarro-Cerrillo, R.M.; Swetnam, T.W.; Zavala, M.A.; 2012c. Is drought the main decline factor at the rear edge of Europe? The case of southern Iberian pine plantations. Forest Ecol. Manag. 271, 158-169. https://doi.org/10.1016/j.foreco.2012.01.040

Sánchez-Salguero, R.; Camarero, J.J.; Dobbertin, M.; Fernández-Cancio, A.; Vilà-Cabrera, A.; Manzanedo, R.D.; Zavala, M.A.; Navarro-Cerrillo, R.M.; 2013. Contrasting vulnerability and resilience to drought-induced decline of densely planted $v s$. natural rear-edge Pinus nigra forests. Forest Ecol. Manag. 310, 956-967. https://doi.org/10.1016/j.foreco.2013.09.050

Sánchez-Salguero, R.; Navarro-Cerrillo, R.M.; 2015. La sequía y la gestión histórica como factores del decaimiento forestal en poblaciones de Pinus sylvestris y P. nigra en el sur peninsular. En: Herrer, A.; y Zavala, M.A.; (eds.) Los Bosques y la Biodiversidad frente al Cam- 
bio Climático: Impactos, Vulnerabilidad y Adaptación en España. Ministerio de Agricultura, Alimentación y Medio Ambiente, Madrid, pp. 271-282.

Schvester, D.; 1970, Desarrollos recientes en el conocimiento de los insectos devastadores del pino marítimo en zona mediterránea francesa. Boletín del Servicio de Plagas 26, 173-179.

Schvester, D.; Ughetto, F.; 1986. Différences de sensibilité à Matsucoccus feytaudi Duc. (Homoptera: Margarodidae) selon les provenances de pin maritime (Pinus pinaster Ait.). Annales des Sciences Forestières 43, 459-474. https://doi.org/10.1051/forest:19860403

Soria, S.; Moreno, M.; Viñuela, E.; Del Estal, P.; 2000. Principales cochinillas en los pinos epañoles. Boletín de Sanidad Vegetal 26 (3), Ministerio de Agricultura, Pesca y Alimentación.

Tavares, C.; Roques, A.; Courtial, B.; Branco, M.; Jactel, H.; Lopez-Vaamonde, C.; 2015. Phylogeography of the ladybird Iberorhyzobius rondensis a potential biological control agent of the invasive alien pine bast scale Matsucoccus feytaudi. BioControl 60, 59-69. https://doi. org/10.1007/s10526-014-9622-7 
